few dynamical variables, in contrast to a microscopic model. Comparison of the results of TDHF models with those of macroscopic models will then be very fruitful in providing a physical insight into the mechanisms involved in the collision, which is difficult to obtain directly from TDHF calculations.

The TDHF equations have been solved, despite their enormous technical difficulty, for a large range of cases going from collisions between s-d shell nuclei to collision of uranium on uranium. One may extract from the results, some general and qualitative features. Fusion cross sections are generally well reproduced, which indicates that at least some features of dissipative processes are correctly taken into account in a mean field description. This is confirmed by the calculation of average kinetic energy loss as a function of deflection angle. However, for low impact parameter and sufficiently high bombarding energy, TDHF always indicates a transparency of each ion to the other. Despite a large kinetic energy loss, the emerging fragments are very similar to the colliding ions. This prediction does not seem to be supported by experiment and is an indication of the deficiency of a simple mean field approximation. In general then, one can say that TDHF seems to predict well the average properties but does not reproduce the dispersion around them.

These conclusions have motivated recent work which tries to understand more deeply what is included in the TDHF approach and the limits of its applicability. It is also searching for modifications that should be made to the TDHF equations. Two important questions suggest themselves:

(1) what is the mechanism that generates the dissipation in TDHF and how does it relate to the classical dissipative formalism which makes a distinction between one and two body viscosity?

(2) what are the effects of two body collisions on TDHF solutions?

The purpose of the Istanbul nuclear physics symposium is to give a general review of the experimental situation as it concerns heavy ion collisions, together with the most recent developments of mean field models and their extrapolations.

\section{Continuation}

Further texts in the series will be published in the February issue of Europhysics News, notably:

High Energy and Particle Physics, 1981: Where Do We Stand? G. Preparata; Trends in Quantum Electronics,

D.S. Chemla;

Physics Education in Secondary Schools in

Europe, G. Born:

Physics and Society at Istanbul,

D.H. Parkinson.

\title{
Recent Developments in Solid State Physics
}

\section{J.T. Devreese, Antwerp}

Considerable progress has been realized in condensed matter physics over the past few years as new techniques have become available on both the experimental and theoretical side. On the experimental side one could name, inter alia, the use of synchrotron radiation, the application of angular resolved photoemission spectroscopy (ARPES), or the extended X-ray absorption fine structure technique (EXAFS). On the theoretical side, the concepts and techniques of the renormalization group, or the ever increasing power of computational techniques to calculate electronic structures come to mind. Also the architecture of materials has developed; e.g. using molecular beam epitaxy, superlattices are prepared with composition and smoothness controlled on the scale of atomic monolayers.

Fundamental insight and technological applications have evolved. New phenomena and new materials emerge. Our understanding has been deepened for such diverse phenomena as melting in two dimensions, or the onset of turbulence in a Rayleigh-Benard experiment in $\mathrm{He}$. The dispersion of surface phonons and of electron energy bands has been determined experimentally for the first time. Wigner localization (in two dimensions) seems to have been observed (Fig. 1) and a superconducting state was discovered in a (quasi one-dimensional) organic conductor under a pressure of $12 \mathrm{kbar}$. A model to describe spin glasses seems to unfold and a "new" state of matter (Fig. 2) (involving bound vortex pairs with opposite vorticity in thin films of ${ }^{4} \mathrm{He}$ ) has been first predicted and then observed. Micro-electronics is one of the predominant fields of successful application; in "submicron devices", soon "man-made" quantum effects should be encountered. Other applications concern opto-electronics or solid state displays.

Condensed matter physics in an extremely vast discipline and emphasis is shifting rapidly as general conferences like the "March Meeting" of the American Physical Society or our own European Condensed Matter Division Conference succeed each other.

At the focus of attention at the 1980 APS March Meeting were photoemission spectroscopy as a probe of electronic band structure, surface probing via EXAFS, tunnelling two-level systems in metallic glasses, surface magnetism, superlattices - the newly designed close packing for computer memories - the physics of onedimensional organic conductors, $\mathrm{CuCl}$ and the diamagnetic anomaly, localization in thin wires and films, space charge layers and Wigner localization, intermediate valence etc. ...

Most of the topics dominating the APS Meeting were also central themes at the Antwerp meeting in April. Nevertheless, in Antwerp the emphasis was somewhat different as can be seen from the report in Europhysics News 11, (1980) 5, p. 7. The creation of a new quantum gas - atomic hydrogen (a little at the border of "condensed" matter) - and nuclear antiferromagnetism or ferromagnetism were two

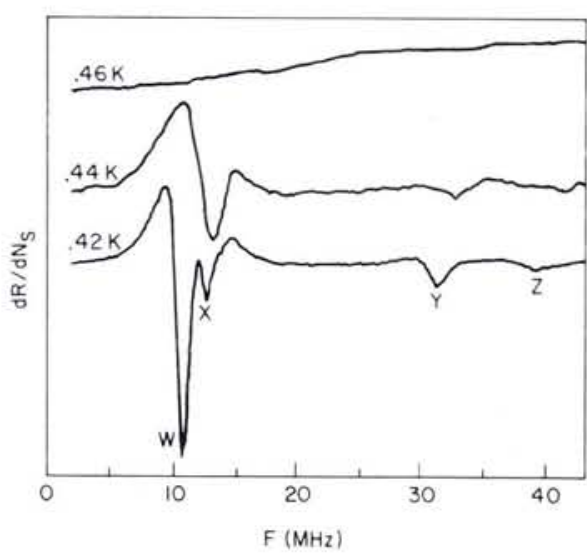

Fig. 1 - Experimental traces displaying the sudden appearance with decreasing temperature of coupled plasmon-ripplon resonances. The resonances only appear below $0.475 \mathrm{~K}$ where the sheet of electrons floating on a liquid helium surface has crystallized into a triangular lattice. (From C.C. Grimes and G. Adams, Phys. Rev. Lett., 42 (1980) 796)

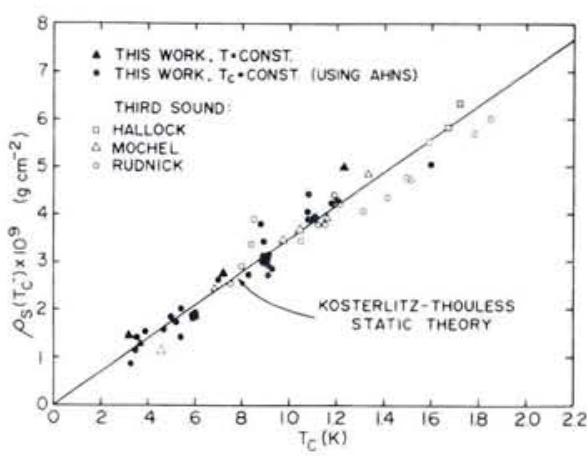

Fig. 2 - Results of the static jump in the superfluid density as a function of the transition temperature. Also included are previous third-sound results. The solid line is the Kosterlitz-Thouless static theory. (From D.J. Bishop and J.D. Reppy, Phys. Rev. Lett., 40 (1978) 1727) 
of the highlights there. Furthermore, topological approaches to covalent and metallic glasses, elementary excitations in topologically disordered materials, the study of the magnetic state of iron above $T$ and nonlinear excitations as also related to biological systems received special attention. Lyotropic liquid crystals, polarons, A15 compounds, first principle phonon calculations, the onset of turbulence, the infinite range model for spin glasses were other central subjects.

\section{Low Dimensional Solids}

Solids with "reduced dimensionality" have continued to catch the imagination of physicists. For a long time considered as merely a textbook exercise, "one-dimensional" (or quasi-one-dimensional) metallic behaviour has attracted much activity since Alan Heeger's observation of an apparently divergent conductivity peak in TTF-TCNQ at $60 \mathrm{~K}$, below which the conductivity falls exponentially to zero as $T \rightarrow 0$. A genuinely superconducting state seems to have been reported now at $T_{\mathrm{c}} \sim 0.9 \mathrm{~K}$ under pressure (> $10 \mathrm{k} \mathrm{bar}$ ) in a derivative of TTF-TCNQ namely (TMTSF) ${ }_{2} \mathrm{PF}_{6}$.

The study of one-dimensional metallic conductivity has evolved more recently into the development of new metallic (linear) polymers. It has been shown that polyacetylene $(\mathrm{CH})_{x^{\prime}}$ a large bandgap semiconductor, can be transformed by doping into an (organic) polymeric metal. The weak interchain interactions in the nearly one-dimensional polyacetylene seem to prevent a metal-insulator transition in the heavily doped polymer. From the applied point of view, rectifying $\mathrm{p}-\mathrm{n}$ junctions and photovoltaic cells have been fabricated from doped polyacetylene; the hope here is to combine metallic and semiconducting properties with the advantages of plastics.

The work on one-dimensional metallic conductors has been - and still is motivated by the search for a high temperature superconductor. Room temperature superconductivity has been the subject of science fiction and scientific dreams for many years. The observation of anomalous high diamagnetism in $\mathrm{CuCl}$ and $\mathrm{CdS}$ should also be noted in this context. The detailed microscopic physical mechanism involved in this giant diamagnetism does not seem to be understood as yet, and there are reports of conflicting measurements and inherent instability. The situation might be comparable to that in the early days of semiconductor physics when the lack of pure, well defined materials confused the picture which is so clear today.

In two-dimensional systems, the emphasis has recently been on phase transitions, particularly on melting. Kosterlitz and Thouless have suggested that melting in two-dimensional systems is a qualitatively different phenomenon from melting in three dimensions. Actual two-dimensional systems have been produced now in the laboratory. In particular it has been possible to form a layer of electrons floating on the surface of liquid $\mathrm{He}$ (at $T<1 \mathrm{~K}$ ). Also in the inversion layer of the $\mathrm{Si}$ metal-oxidesilicon field effect transistor, a two dimensional electron gas has been created. The density and temperature of these twodimensional systems can be tuned easily. For the first time it then becomes possible to detect experimentally a Wigner transition (below $T_{c}$, a triangular electron lattice seems to originate). Studies in this field might also contribute to the understanding of freezing and melting in the "ordinary" (three-dimensional) world.

Systems with reduced dimensionality give rise to a variety of fascinating physical phenomena including instabilities $\left(2 \mathrm{k}_{\mathrm{F}}\right.$ anomaly, see Fig. 3), charge- and spindensity waves, phase transitions, nonlinear solutions (solitons, ...), etc.

Magnetic systems also offer an attractive background to study the effects of reduced dimensionality.

Another phenomenon of current interest is localization in low-dimensional solids. This work relates to the question of the distinction between localized electron states and extended electron states. Mott and Twose had shown that all the electronic states of a disordered one-dimensional system should be spatially localized. The more recent suggestion of Thouless that all systems, regardless of their cross-sectional area, will behave as one-dimensional if they are sufficiently long made the Mott-Twose prediction accessible to experiment. It then follows that wires with impurity resistance larger than $\sim 10 \mathrm{k} \Omega$ would be characterized by thermally activated conductivity at low temperatures i.e. they would be insulators at low temperature. Experiments now seem to confirm the above picture (although the magnitude of the effect is smaller than predicted).

A similar effect is now believed to occur in two dimensions also; electrons in twodimensional systems would always be localized. Although an alternative explanation has been presented, a rise in resistivity at low temperature in thin "metallic" films has been attributed to such localization.

\section{Surface Physics}

In the very broad field of surface physics, attention may be drawn to the following developments:

- the considerable progress in the spectroscopy of surface vibrations. Molecular beams work for surface excitations like neutrons do for bulk phonons and, for the first time, surface phonon dispersion has been measured. This development should be seen against the abundant technological applications of surface phonons (delay lines, signal processing devices, opto-

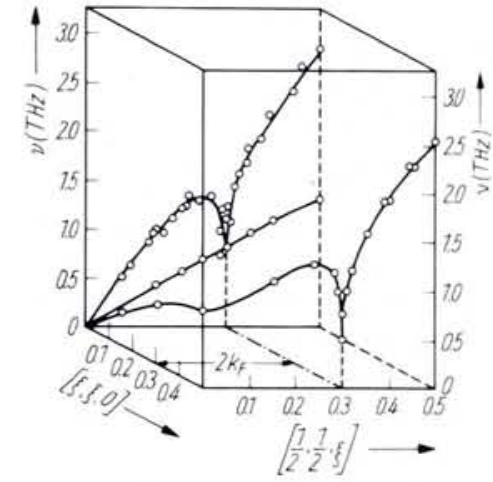

Fig. 3 - Room-temperature dispersion surface of KCP for acoustic phonons propagating along the chain direction. The giant $2 k_{F}$ anomaly is clearly visible for the longitudinal modes. (From R. Comès and G. Shirane, in "Highly Conduc ting One-Dimensional Solids" (Plenum Publ. Corp., New York) 1979, p. 33)

acoustic devices etc. ...). The controlled growth of ideal or stepped surfaces is today a relatively easy matter and the development of signal processing devices in the $\mathrm{TH}$ region is now at hand

- the knowledge of surface phonons and in particular surface soft modes is also required to understand the different surface phases and related surface reconstruction phenomena which are of importance in the physics of gas-surface interaction.

- with the use of high-intensity monochromatic synchrotron radiation along with electron yield detection techniques one now is able to perform EXAFS (see below) measurements on surfaces. These provide i.a. bond lengths of molecules (currently with a precision of 0.02-0.05 $\AA$ ) adsorbed on surfaces. Similar experimental progress combined with computational progress on electronic structures and bonds at surfaces, results in increasing understanding of surface reactions, and other dynamic processes at surfaces including heterogeneous catalysis. Other topics of interest include phase transitions in chemisorbed layers (e.g. the work of A. Malshukov) and giant Raman scattering by molecules in metals.

\section{Amorphous Materials}

Amorphous solids remain a field of major activity.

The nature of the spin glass state is still an unsolved statistical mechanics problem. G. Toulouse has pointed out some surprising features (e.g. negative entropy) of the infinite-range model (a mean field-like description) used to describe the spin glass state while recent experimental work seems to suggest a distinction between "reversible" and "irreversible" magnetization in spin glasses.

The mechanism of conductivity in amorphous metals has recently been connected 
to the conduction electrons and the twolevel defects which seem to be present in all glasses.

The possible use of hydrogenated amorphous $\mathrm{Si}$ in xerography, photovoltaic cells, and vidicons has led to a renewed interest in the structure of amorphous $\mathrm{Si}$ (a-Si) and specifically in hydrogenated amorphous silicon (a-Si:H).

In conventional amorphous silicon (i.e. without hydrogen) the presence of "dangling bonds" prevents the observation of a number of semiconductor phenomena such as photoluminescence and photoconductivity. Also doping is prohibited by those dangling bonds. It has been shown that in hydrogenated amorphous silicon on the other hand, the dangling bonds can be deactivated; photoconductivity and doping become possible again and also p-n junctions can be made.

The physical properties of a-Si:H are determined not only by the hydrogen concentration; the interrelation between the chemical bonding of hydrogen and the morphology is crucial.

\section{Semimagnetic Semiconductors}

Over the past few years it has become possible to prepare alloys of semiconducting compounds and magnetic semiconductors. They are semiconducting mixed crystals containing controlled amounts of magnetic ions and have been named semimagnetic semiconductors (SMSC). Examples are $\mathrm{Hg}_{1-\mathrm{x}} \mathrm{Mn}_{\mathrm{x}} \mathrm{Te}$ or $\mathrm{Cd}_{1-\mathrm{x}} \mathrm{Mn}_{\mathrm{x}} \mathrm{Te}$.

One of the attractive features of SMSC's is the possibility of tuning the semiconducting properties (like conductivity or optical properties) by changing the subsystems of magnetic ions, e.g. by applying an external magnetic field. Alternatively the interactions within the magnetic ion system can be probed experimentally by varying the dilution of the magnetic ions in a semiconducting system of precisely known bandstructure. SMSC's are therefore of fundamental interest, both from the point of view of magnetism and from the point of view of semiconductor physics.

Among the effects observed in SMSC's are very high effective $\mathrm{g}$-factors of band electrons (due to the exchange interaction of band electrons with the electrons of the $3 d^{5}-$ shell of $\mathrm{Mn}^{2+}$ ) and giant Faraday rotation.

SMSC's will be reviewed in Istanbul in the Physics of Semiconductor Alloys Symposium.

Spectroscopy of Solids; Excitons and Polarons

Excitons remain a fascinating subject of solid state spectroscopy. Several types of excitons (trapped and free excitons, Fresnel and Wannier type) and their optical and magneto-optical properties remain the subject of intense investigations. Studies

\section{NATO Advanced Study Institute on \\ "Nonlinear Phenomena at Phase Transitions and Instabilities"}

Manifestations of nonlinearities through experiments and theories for structural phase transitions, hydrodynamical instabilities, crystal growth and low-dimensional melting.

Open to participants from all countries.

Deadline for application 2 February 1981.

Information: Gerd Jarrett, Institute for Energy Technology

POB 40, N-2007 Kjeller, Norway

of electron (hole)-phonon interaction, exchange interaction, involvement of excitons in non-local properties of semiconductors help us to probe the nature of several fundamental interactions in solids. The physics of excitons has influenced the understanding of phenomena in related but different fields: luminescence, photoconductivity, bandstructure.

The nature of bound exciton complexes is still under discussion. Self-trapped excitons, bi-excitons, condensation of excitons, electron hole drops in semiconductors, non-radiative recombination of excitons, nonlinear effects and polariton dispersion are a few topics of current interest.

A "European Spectroscopy group", created on the initiative of S. Nikitine, has met since 1975 twice a year to discuss subjects mostly related to the spectroscopy of excitons.

Although considerable theoretical and experimental attention has been devoted to polarons, several problems remain open; e.g. no satisfactory theory for the hot polaron conductivity (except for small electron-phonon coupling) is available yet, and trat.sport under crossed electric and magnetic fields for intermediate coupling is not well understood either.

Recent progress includes studies of the streaming motion characterized by a highly anisotropic velocity distribution of polarons in $\mathrm{AgBr}$ and $\mathrm{AgCl}$. Somewhat surprisingly the Boltzmann equation seems to account for the observed effects.

\section{Angular Resolved Photoemission \\ Spectroscopy}

One of the several fascinating techniques which have been developed in solid state science over the last decade is angular resolved photoemission spectroscopy (ARPES). By analyzing the angular distribution of the photoemitted electrons one can now probe the dispersion of the energy levels of electrons in solids just as by using inelastic neutron scattering the phonon dispersion curves can be measured. Another analogy is the experimental deter-

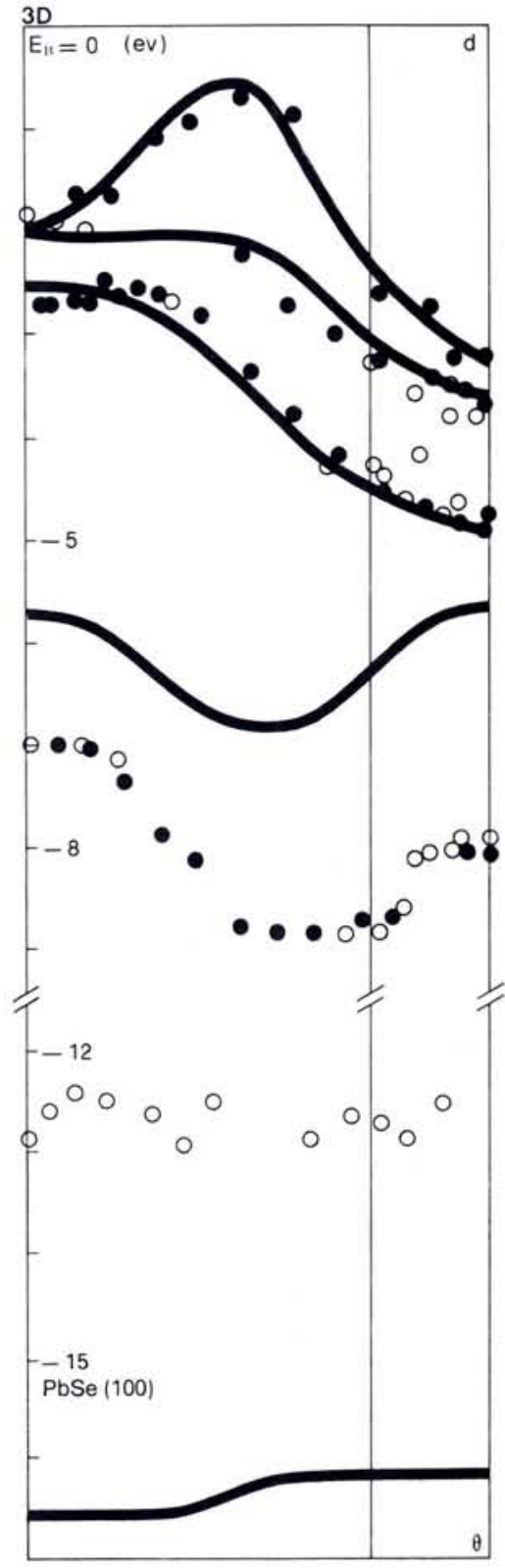

Fig. 4 - Bandstructure of the three-dimensional semiconductor PbSe. The curves obtained from a pseudopotential calculation agree very well with the experimental data $\left(^{\circ}\right.$ and ${ }^{\bullet}$ for p-like bands. Experiment also shows the weakness of the calculation for s-like bands. (From Y. Petroff, La Récherche 9 (1978) 477) 
mination of plasmon dispersion curves, which is of importance for a deeper understanding of correlations in many body systems.

ARPES provides us with "experimental bandstructures" which are so central to the single particle picture of solids. For many years, theorists have been providing bandstructure calculations which could only be compared to experiment in a few symmetry points. This is a particularly fortunate circumstance: all these theoretical bandstructures provided in tempore non suspecto, when theorists did not consider even the remote possibility of energy vs. wave number measurements, can now be compared to ARPES experimental data. Whenever theory and experiment do not fit, one may learn something new about the electronic structure in solids or about the shortcomings of pseudopotentials, OPW, etc. ... Pseudopotentials seem to work rather well in several compound semiconductors (including GaAs) where ARPES has been applied. In the case of $\mathrm{PbSe}$, some discrepancies between pseudopotential theory and experiment occur (see Fig. 4).

Whereas the available band theory works very well for $\mathrm{Cu}$, some experimental splittings in the band structure of $\mathrm{Ni}$ do not coincide with experiment at all. Large electron-electron correlations related to the magnetic properties might be responsible.

ARPES measurements are now commonly performed using synchrotron radiation, which increases their general utility.

\section{Synchrotron Radiation}

The use of synchrotron radiation has already been mentioned in the context of EXAPS studies at surfaces and ARPES measurements of band structures. Synchrotron radiation, "the light of the future", with its white X-ray spectrum and unprecedented intensity, has many other applications including X-ray lithography, $\mathrm{X}$-ray diffraction topography etc. ... A particularly nice application is the use of anomalous $\mathrm{X}$-ray scattering to solve the socalled phase problem for crystal structure of proteins or other large molecules. Anomalous scattering occurs when $\mathrm{X}$-rays have energies close to that required to ionize an inner electron. Because of Friedel's law, the scattering amplitudes $F(h, k, 1)$ for a particular Bragg reflection (h, k, I are the "indices" of the Bragg reflection) satisfy $|F(\bar{h} \bar{k} \backslash)|^{2}=|F(h k \mid)|^{2}$ and therefore phase information is lost.

Using the tunability of the synchrotron radiation and measuring diffraction patterns at various wavelengths close to the absorption edge, the structure factor F (h k l) | can be changed so as to break Friedel's rule, and thus provide information on the phases

It may also be noted that dynamic studies of biological matter have become

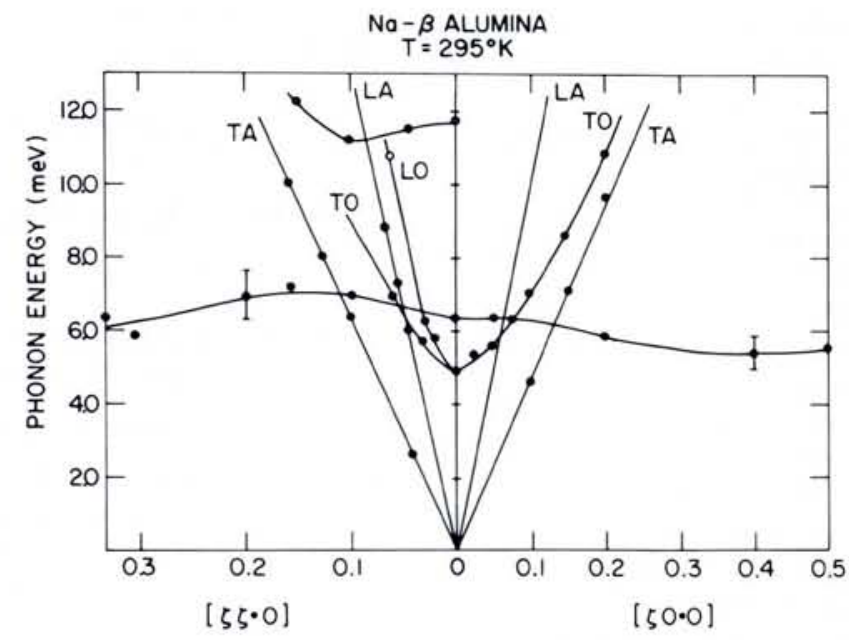

Fig. 5 - Phonon dispersion curves for Na-beta alumina. The liquid "component" gives rise to the almost dispersionless mode at ca. 6 meV. (From S.M. Shapiro, in "Superionic Conductors" (Plenum Publ. Corp., New York) 1976, p. 271)

feasible with synchrotron radiation fibre diffraction.

Several synchrotron radiation centres are (or will be) available in Europe and European physicists should be able to play a major role in future developments.

\section{Fast Ion Conduction}

Research on superionic conductors has been stimulated by the interest in developing alternative energy sources, in particular renewable sources, e.g. for use in transportation. What is needed is storage of electrical energy of sufficiently high density to allow the propulsion of vehicles by electric power. Conventional storage batteries with liquid electrolytes are not practicable whereas solid electrolytes can have conductivities (associated with ionic motion) comparable to those of liquid electrolytes. Normally the ionic conductivity in solids is very low; however some solids do show relatively high conductivities and these are called "superionic conductors" (their ionic conductances exceed $0.01 \mathrm{ohm}^{-1} \mathrm{~cm}^{-1}$ ). Examples are beta alumina, silver iodides, anion conductors.

The high conductance of the superionic conductors is associated with one ion species diffusing away from its normal lattice position and is associated with lattice disorder. Extensive fundamental explorations have been devoted to the conduction mechanism and the dynamics of the disorder. Superionic conductors provide an interesting state of matter in which a liquid of ions (e.g. $\mathrm{Ag}^{+}$in $\mathrm{Agl}$ ) is enclosed in a lattice formed by other ions (the $\mathrm{I}^{+}$ions in Agl) (see Fig. 5). Of fundamental interest is the nature of the phase transition (from a poorly conducting to a superionic state) occurring in superionic materials as a function of temperature.

The Istanbul programme contains several papers devoted to the ionic conductivity mechanism.

\section{Very Large Scale Integration Circuits and Materials}

Obviously very large scale integration is one of the major lines of development in applied physics, and microscience is the new term coined to describe the research on the scale of the microscopic structures that are now available. Microscience is, to some extent, a new science; the relevant size of the systems is now $100-1000 \AA$ and fundamental questions arise at this quantum level where several textbook idealizations of solid state physics (e.g. continuous energy bands) reach their limits of applicability. Microelectronics with its everincreasing capability of producing microstructures with 60000 (and more) transistors on one chip, with its refined and new processing techniques (epitaxial growth of crystal layers, optical lithography, ion implantation and more recently laser or electron-beam annealing, graphoepitaxy, molecular beam epitaxy ...) with its many applications (computer systems, clocks, analog-to-digital transducers, self calibrating instruments and a thousand other applications) now enters the submicron device area where the prospect is the fabrication of solid-state logic circuits at "biological" densities. A new industrial revolution is forecast.

Substantial cross fertilization between applied and fundamental research in microscience is taking place, with superlattices at the centre of activity. Superlattices are an example of new materials made possible by microfabrication. A layer of $\mathrm{GaAs}$ e.g., about $100 \AA$ thick, is sandwiched between confining layers of $\mathrm{Al}_{\mathrm{x}} \mathrm{Ga}_{1-\mathrm{x}}$ As which may be doped. These sandwiches are expanded to hundreds of $\mathrm{GaAs}$ layers with interspersed $\mathrm{Al}_{\mathrm{x}} \mathrm{Ga}_{1-\mathrm{x}} \mathrm{As}$ and a superlattice, with periodicity 10 or 100 times the lattice parameter, is created.

These superlattices are said to exhibit man-made quantum size effects. The conduction electrons have a tendency to con- 
centrate in the GaAs layers where their mobility can be considerably larger than in bulk GaAs. The mobility in the layers is larger than in Si, leading to the prospect of higher switching speed than in conventional devices. Not only are superlattices candidates for the further development of miniaturization, they also reveal many (new) physical phenomena occurring at the submicron level. The quantum localization in thin wires and films discussed above constitutes one example; the resistivity of the thin wires (measurements on wires with diameters as small as $300 \AA$ have been performed) is no longer proportional to the length of the wire (as in the microscopic case) but grows exponentially as a function of length.

Other physical phenomena observed in superlattices include the semiconductorsemimetal transition in InAs-GaSb superlattices, which occurs with increasing layer thickness and manifests itself as a sharp rise in carrier concentration. This transition occurs at a layer thickness of about $100 \AA$. Investigations have also been devoted to the study of cyclotron resonance, Hall effect and magnetic properties of the twodimensional electron gas confined in the GaAs layer.

In Istanbul, in addition to the plenary invited talks on VLSI and "The Impact of Microelectronics", there will be sessions on ion implantation and laser annealing.

\section{Other Vital Topics}

It is quite impossible to treat the recent developments in a field as vast as solid state physics in any detail in one article. In what follows, I simply mention a few topics of current interest,

The application of modern physical concepts to biology

\section{Spin effects and impurities}

The use of muons in solid state physics

Convective instabilities and the onset of turbulence

Local field effects in semiconductors

Superconductivity

\section{Some Omitted Trends}

Fundamental research:
- the extension of neutron inelastic scattering to energies
larger than $0.1 \mathrm{eV}$
- mixed valence
- atomic and electronic structures of small aggregates
- n photon excitations in crystals
- magnetism in metallic and non-metallic substances
- time resolved spectroscopy, picosecond spectroscopy in the
optical region, nanosecond spectroscopy in spin resonance
- the calculation of radiation pressure as derived from micro-
scopic electromagnetic theory and statistical mechanics so as
to obtain reliable macroscopic expressions
Applied research:
- new superconductivity alloys
- developments in integrated optoelectronics (optical fibre
communications)
- plasticity of metglasses and ionocovalent solids
- recrystallisation kinetics of polymers
- solid state displays
- Josephson junctions
- power devices
- metal-semiconductor contact devices
- light emitting devices
- amorphous soft magnetic materials, hard magnetic materials,
etc. ...

undamental research:

the extension of neutron inelastic scattering to energies

atomic and electronic structures of small aggregates $n$ photon excitations in crystals

magnetism in metallic and non-metallic substances

resolved spectroscopy picosecond spectroscopy in the the calculation of radiation pressure as derived from micro tistical mechanics so as

(optical fibre

plasticity of metglasses and ionocovalent solids

tics of polymers

light emitting devices

etc.

\section{What's New \\ in Computational Physics}

\section{F. James, CERN}

As computers become more versatile, faster, and more easily accessible, so do the applications of computers to physics research become more varied and important each year. This is nothing new; it has been true ever since the use of computers in physics became widespread about twenty years ago.

The amazing thing is that it remains true despite the constant acceleration of progress in computing technology. Current advances are so great that one can really talk about qualitative changes in the way computers are used (even perhaps changes in the definition of a computer!), and we are now led to speculation as to when the acceleration will come to an end. Indeed only in a few areas are we approaching known physical limits; in all other domains there is still no known barrier to progress.

A striking example of the effects of technological progress was offered recently at the Europhysics Conference on Computing in High Energy and Nuclear Physics, held in Bologna in September, 1980 (Europhysics Conference Abstracts, Vol. 4G, Europhysics News, 11 (1980) 10, p. 6). In the session devoted to online data processing, a series of 12 contributed papers described highly sophisticated systems, based on both microprocessors and minicomputers, driving vast heterogeneous arrays of detectors, making complex triggering decisions, filtering and recording data at megabit rates. What made these presentations especially impressive was that for the most part they were describing not plans for the future, but real experiments already taking data.

At the same conference it was pointed out that the revolution in microcircuitry has not yet made its main impact on large computer systems, and that when it comes, central computing facilities will be even more in demand than now, in spite of some gloomy predictions about their declining role. Certainly the astronomical complexity of nuclear physics calculations, far from making the large computer obsolete, are ready to absorb all the increased capacity that the new technology can supply.

\section{Magic of VLSI}

Of course the magic component in all this is VLSI - the Very Large Scale Integration of thousands of circuit elements on to one chip, with the resulting possibilities of enormously increased speed and capacity of logical and arithmetic operations and data storage. This will be one of the major topics to be treated at the EPS General Conference in Istanbul. Plenary sessions and studies organized by the Physics and
Society Advisory Committee will allow both the technical and social implications to be explored. These should be of great interest to all physicists. VLSI technology brings us not only new dimensions in speed and capacity, but through modularity and microprogrammability, unprecedented flexibility and reliability.

But the price we have to pay for all this, at least temporarily, is that the most modern devices are delivered naked, and so we find ourselves interfacing and programming those wonderful chips at the very lowest level, sometimes even altering or extending their basic instruction set through microcode. This situation, reminiscent of the early days of large computers, discourages even some experts from getting too involved in microprocessors now. (One eminent computer physicist has remarked: "I enjoy studying history, but I am reluctant to relive it".) On the other hand, it is this very aspect which fascinates many others. In any case, no matter at what level you interact with the new technology, it can no longer be ignored.

\section{Memory Systems}

Progress in magnetic storage has also exerted a great influence on the use of computers in physics. At one end of the scale, the widespread use of floppy disks has made this technology accessible to even the most modest computer system, and at the other end of the scale, advances in high-capacity disks have been steady and spectacular over several years. The capacity, transfer rates, access times, reliability, and local "intelligence" of disk units have all made dramatic increases in recent years, all of which have had a significant impact on computer systems.

A very exciting recent breakthrough, still being developed into a practical device, is the ability to record data optically by means of laser-drilled spots of less than 1 micron diameter, giving the possibility of recording (once-only, of course) nearly $10^{10}$ bits on a single $30 \mathrm{~cm}$ diameter disk surface. Reading can be achieved with arbitrarily low error rate, access time of $50 \mathrm{~ms}$, and transfer rate of 10 megabits/s. We look forward to mass-storage devices based on this technology which should be commercially available in the very near future.

\section{Communications}

In the area of data communications, networking is finally becoming common in Europe, mostly on a local scale, but also to some extent internationally. At the Bologna conference, we heard of the problems in international data transmission, 